\title{
Polysèmes
}

Revue d'études intertextuelles et intermédiales

\section{À la recherche du lien et du moment perdus : « 57 Dartmouth Square » et «Il Bambino Dormiente » de Paul Durcan}

Looking for the Lost Link and the Lost Moment: "57 Dartmouth Square" and "Il Bambino Dormiente" by Paul Durcan

\section{Cathy Roche-Liger}

\section{OpenEdition}

\section{Journals}

Édition électronique

URL : http://journals.openedition.org/polysemes/2289

DOI : 10.4000/polysemes.2289

ISSN : 2496-4212

Éditeur

SAIT

\section{Référence électronique}

Cathy Roche-Liger, « $\grave{A}$ la recherche du lien et du moment perdus : « 57 Dartmouth Square » et « II Bambino Dormiente » de Paul Durcan », Polysèmes [En ligne], 18| 2017, mis en ligne le 30 novembre 2017, consulté le 20 avril 2019. URL : http://journals.openedition.org/polysemes/2289 ; DOI : $10.4000 /$ polysemes.2289

Ce document a été généré automatiquement le 20 avril 2019.

Polysèmes 


\title{
À la recherche du lien et du moment perdus : « 57 Dartmouth Square » et «Il Bambino Dormiente » de Paul Durcan
}

\author{
Looking for the Lost Link and the Lost Moment: "57 Dartmouth Square" and "Il \\ Bambino Dormiente" by Paul Durcan
}

Cathy Roche-Liger

1 Dans The Days of Surprise (2015, ci-après DS), ouvrage à forte dimension autobiographique, le poète irlandais Paul Durcan capture et fixe des souvenirs, et semble ainsi créer un album à la fois composé d'instantanés et de portraits. C'est un de ces instantanés que le poète transcrit dans «A Charm of Goldfinches » (31-32), lorsqu'il se décrit dans un pub en compagnie d'un ami qui décide, sous l'impulsion du moment, de libérer des chardonnerets de leur cage. Ces oiseaux sont en plein vol sur la couverture du recueil qui est un détail de la peinture Goldfinches de David Inshaw. Ce sont quatre détails de cette même œuvre, montrant chacun un chardonneret à différents instants de son vol, qui font office de repères visuels marquant le début du recueil et les séparations entre les différentes parties. Le vol de ces oiseaux peut être vu comme un symbole de ces moments de surprise qui parcourent la vie et viennent s'inscrire dans le titre du recueil et du poème éponyme. Leur envol peut être aussi perçu comme l'impulsion poétique, et le chardonneret comme une incarnation du poète, de son chant et de son parcours en cette vie. Leur capture en plein vol fait écho à la volonté durcanienne de saisir des moments passés et de les fixer dans ses vers, tout comme une photographie peut saisir un mouvement au vol et semble ainsi suspendre le temps. C'est ce que Durcan exprime lors d'un entretien lorsqu'il déclare, à propos du poème rock intermédial « The Poet and the Judge ", lui-même extrait de The Days of Surprise : «I'm trying to record, in verse, a momentlike a photograph-things that happened» (Wallace, nous soulignons). De surcroît, dans les représentations de Vierge au chardonneret, variations du thème de la Vierge à l'Enfant, le 
chardonneret sert à annoncer la Passion et la couronne d'épines, par l'entremise du chardon $^{1}$ dont il se nourrit et auquel son nom latin renvoie, ainsi que par le rouge de la tache qui orne sa tête et évoque le sang du sacrifice. Cet élément de l'iconographie chrétienne vient renforcer le lien qui semble unir le poète à cet oiseau puisque Durcan s'identifie au Christ de manière répétée : à travers « 57 Dartmouth Square » (3-8) et «Il Bambino Dormiente » (26-28), The Days of Surprise ou son œuvre en général. En outre, le poète inscrit en exergue de The Days of Surprise une citation de Helen Waddell extraite de The Wandering Scholars, ouvrage qui se fait plaidoyer contre l'oubli de l'œuvre des poètes médiévaux anonymes ou itinérants dont il retrace l'histoire : «On these the iniquity of oblivion hath blindly scattered her poppy ». Par ce choix, il manifeste sa peur de l'oubli et son désir de faire de son recueil un rempart contre la mort et le néant, un écrin enchanté où il placerait et préserverait des souvenirs, des portraits d'inconnus ou d'êtres chers. Dans The Days of Surprise, où son catholicisme et sa foi en la résurrection se font entendre plus nettement que précédemment, Durcan écrit la vie et toute la variété des émotions humaines: ses maladies, ses deuils et ses moments de tristesse, de colère, voire d'indignation, mais aussi ses moments de surprise et de joie. Les vers suivants, qui décrivent Ulysses de Joyce et sont extraits du poème élégiaque à la mémoire d'Elizabeth Walsh Peavoy, pourraient ainsi être considérés comme une définition de la poésie durcanienne et comme un résumé à la fois de la diversité et de l'unité de The Days of Surprise et des 67 poèmes qui le composent : « But of course [...] you have to remember / That Ulysses is poetry- / It's what's called the Panoply of Human Experience- / A lot of it may never have happened " (nous soulignons, DS, 120). Dans The Days of Surprise, Durcan tisse souvenirs, faits d'actualité et rêveries fantasques pour créer cette "Panoplie de l'Expérience Humaine ». Comment le poète esthétise-t-il ces moments envolés ? À quelles images a-t-il recours ou comment parvient-il à «faire image " pour reprendre l'expression de Jean-Luc Nancy et ainsi à « donner du relief, du saillant, du trait, de la présence » à des instants passés (Nancy 2003, 125) ? Comment crée-t-il, par le biais de ces images, des espaces où différentes temporalités sont condensées : celles du mythe et de l'histoire ; celles de l'avoir-été, de l'être et du devenir? En nous concentrant d'abord sur la lecture de « 57 Dartmouth Square » (DS, 3-8), nous décrirons, à travers son jeu sur des moments d'apothéose et d'épiphanie, son fantasme d'un paradis perdu, son refus de la séparation d'avec la mère, son besoin de repères, ses interrogations sur la nature de son être ainsi que sur sa place dans le monde. Nous soulignerons comment sa quête d'un sentiment d'appartenance et son impossible deuil du lien à la mère s'écrivent entre immobilité et mouvement, intérieur et extérieur, stabilité et instabilité. Nous verrons ensuite comment cette quête et son désir du retour à l'enfance aux côtés de la mère s'écrivent de manière répétée : comment ils se propagent intratextuellement de recueil en recueil et à travers The Days of Surprise, et notamment au sein de «Il Bambino Dormiente » (26-28), poème où la résurgence du passé aboutit à une forme de réconciliation avec le présent et l'avenir.

\section{« 57 Dartmouth Square »: (ré)écriture de moments d'épiphanie et de l'Épiphanie}

2 Dans « 57 Dartmouth Square », le long poème de six pages par lequel s'ouvre The Days of Surprise, Paul Durcan revient à ses racines, à sa première adresse à Dublin, sur le Grand 
Canal, et se dépeint, en lien avec ce lieu, à des moments clefs de sa vie qu'il qualifie de moments d'apothéose et d'épiphanie. Bachelard écrit :

On croit parfois se connaître dans le temps, alors qu'on ne connaît qu'une suite de fixations dans des espaces de la stabilité de l'être, d'un être qui ne veut pas s'écouler, qui, dans le passé même quand il va à la recherche du temps perdu, veut "suspendre " le vol du temps. Dans ses mille alvéoles, l'espace tient du temps comprimé. L'espace sert à cela. [...] C'est par l'espace, c'est dans l'espace que nous trouvons les beaux fossiles de durée concrétisés par de longs séjours. L'inconscient séjourne. Les souvenirs sont immobiles, d'autant plus solides qu'ils sont mieux spatialisés. (27-28)

Ici, le poète rêve à la maison de son enfance, à un espace constitutif, et c'est en tissant souvenirs et images de cette maison et de son environnement proche qu'il s'autoreprésente à diverses reprises. Oscillant au fil des strophes entre être et devenir, immobilité et mouvement, Durcan se cherche et «aspire à être " par l'écriture, pour reprendre l'expression par laquelle le texte s'achève («I aspired to be », DS, 8). D’un côté, par l'identification initiale répétée avec la maison, le texte apparaît comme la recherche d'un « espace de la stabilité de l'être » (Bachelard, 27), de la conscience d'être. De l'autre, par l'entremise du processus d'identifications changeantes, qui peuvent être lues comme l'expression d'un déficit d'être, le texte se fait quête de la place du poète dans le monde. Cette oscillation entre être et devenir, stabilité et instabilité, immobilité et mouvement est renforcée tout au long du poème par une dialectique du dedans et du dehors, de l'entrée et de la sortie (notamment par la répétition des prépositions « in and out », 4), de l'intérieur et de l'extérieur, de l'espace clos et ouvert.

Dans la première partie de ce texte, Durcan choisit de recourir à une forme de syncrétisme primitif lorsqu'il se décrit sous les traits de sa maison d'enfance, c'est-à-dire comme fusionnant avec son environnement, sans qu'il y ait distinction entre lui et la maison:

I was three years of age in the full of my days,

Never again to be so fully myself.

I was my home, my home was my name-

57 Dartmouth Square.

All that I was, now and for ever,

Today, yesterday and tomorrow,

57 Dartmouth Square.

Sometimes I was called Paul

But mostly I was not a who or a what

But a where.

I was a place. [...]

I was a three-storey, red-brick terraced house [...] (3-4)

Par le biais de cette indifférenciation primitive, il crée son propre mythe de l'origine, son centre du monde, son point fixe.

5 «Quand on rêve à la maison natale, dans l'extrême profondeur de la rêverie, on participe à cette chaleur première, à cette matière bien tempérée du paradis matériel » (Bachelard, 27). Cette rêverie ramène, selon Bachelard, à un instant où « l'être est l'être-bien ", à « la plénitude première de l'être de la maison » (25-27), et c'est bien cette plénitude qui est initialement décrite (" in the full of my days », «be so fully myself », DS, 3). Cet espacetemps de bonheur premier est qualifié d'apothéose: «I would never again know such apotheosis / As the place that I was, 1947-1948 » (3). C'est un moment d'intensité qui, bien que délimité, se prolonge et s'inscrit dans la durée : «I was three years of age », «a long 
time », «the winter of 1947-48» $(3,4,6)$. Ici, l'apothéose n'est pas une apogée, une conclusion brillante, mais un quasi-commencement, qu'il situe à l'âge de trois ans. C'est dans un paradis terrestre, localisable sur une carte, en ce lieu « 57 Dartmouth Square » ( $D S, 3)$, que le poète a ressenti une telle félicité. Cette plénitude est placée du côté du mythe et se teinte de Christianisme en étant associée au paradis ( "Heaven ») :

I was three years of age in the full of my days,

Never again to be so fully myself. [...]

Heaven was a place-not a placeless heaven-

And I was that place-

57 Dartmouth square. (3)

6 C'est comme s'il avait vécu en cette vie un temps prélapsarien avant de chuter à son tour, en cessant d'être ce lieu : « One day I'd cease to be 57 Dartmouth Square » (5). Cependant, ce moment est présenté de manière paradoxale comme à la fois dans l'histoire et dans le mythe, puisqu'il est défini au sein d'une chronologie personnelle, dans une linéarité historique, à un moment donné et délimité (l'hiver 1947-48) tout en étant placé hors du temps :

All that I was, now and for ever,

Today, yesterday and tomorrow,

57 Dartmouth Square. (3)

Cette félicité extrême est liée à un sentiment d'appartenance au monde, de présence : « In the winter of 1947-1948 holding Mummy's hand, / Knowing where I was, I'd reached already the Promised Land» (6). Ainsi, c'est un espace de bonheur premier, de l'intimité, où il ne fait qu'un avec la maison et sa mère ; c'est un espace qui peut s'étendre au dehors, lors de balades, à condition qu'il tienne la main de sa mère («Holding hands with Mummy », DS, 5). H. U. Gombrecht insiste sur la dimension spatiale de la présence ("The word "presence" does not refer (at least not mainly refer) to a temporal but to a spatial relationship to the world and its objects ", xiii). En s'associant à un objet tangible du monde, à un lieu fixe, à une maison, Durcan insiste sur ce sentiment de présence éprouvé autrefois. L'espace poétique est à la fois le lieu où il exprime sa quête d'un paradis (terrestre) perdu et où il opère une forme de résurrection du passé. Il rend celui-ci à nouveau présent en répétant cette adresse et cette date comme une litanie, une invocation, mais aussi en décrivant précisément sa localisation, ses origines, ainsi que son aspect:

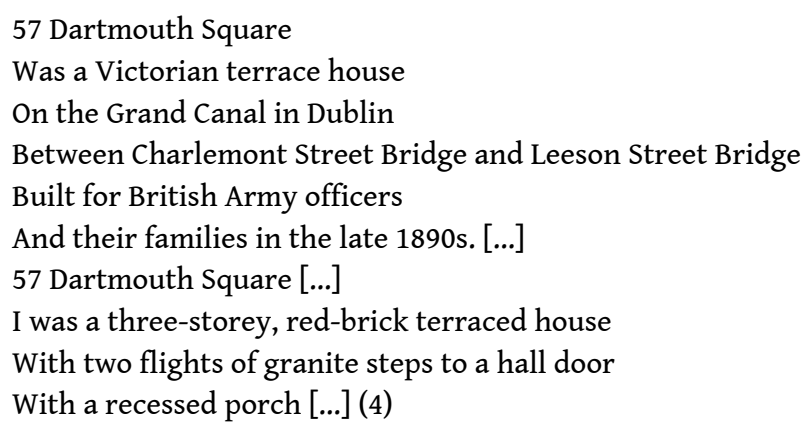

Comme tout moment d'intensité et tout effet de présence, ce temps d'apothéose et de plénitude est éphémère, comme le souligne le poète: «One Day I'd cease to be 57 Dartmouth Square » (5). Cette "Terre Promise» («the Promised Land», 6) n'est pas ce qu'il cherche mais ce qu'il a quitté. Un renversement est opéré et une sorte d'exode s'ensuit. En effet, la plénitude première fait place à «la métaphysique consciente qui se place à l'instant où l'être est "jeté dans le monde" » (Bachelard, 26). Au fil de la rêverie 
poétique, elle s'actualise de façons diverses. Ainsi, dans les strophes 10 et 11, Durcan se place au moment où il fait l'expérience de "l'hostilité des hommes et de l'univers" (Bachelard, 26). Cependant, il n'y a pas simple opposition entre une plénitude première désormais inaccessible - et l'hostilité de l'univers. Cette plénitude fait aussi place à l'extraversion, ou du moins au désir d'une rencontre avec l'extérieur. Elle peut prendre la forme passive et inversée d'une attente de l'autre («The remainder of my life I would spend / Waiting for the Three Wise Men to find me ", DS, 6, strophe 8). Elle se double aussi d'une sorte de "rêverie du chemin » (Bachelard, 29, qui s'actualise ici par une course, DS, 6 , strophe 9) et d'ouverture au monde (DS, 8, strophe 12).

Durcan fait de l'espace de la maison un lieu nodal qui concentre différentes temporalités : l'histoire et le mythe ; l'avoir-été, l'être et le devenir. C'est par ce lieu qu'il se définit et c'est à partir de ce point fixe qu'il (re)trace son évolution à travers une série d'autoportraits et de souvenirs.

Il commence par complexifier son autoportrait sous les traits de la maison en incorporant une deuxième image dont la dimension picturale est renforcée par les indications de couleurs («red», « white», "grey», 3). Il se désigne par le biais d'une étiquette qui a d'autant plus de pouvoir de nomination qu'elle est inscrite sur son corps, brodée d'un fil rouge sur un vêtement porté, ses chaussettes :

I answered to the name of Paul [...]

But knowing that my real name,

My identity tag stitched in red thread

On white cotton on my grey socks,

Was 57 Dartmouth Square. (3)

11 Cette image crée elle aussi une condensation de temps, puisqu'elle ramasse et cristallise les deux pôles opposés de la vie: cette étiquette évoque dans l'imaginaire du lecteur le nom brodé par la mère sur les vêtements d'un enfant et l'étiquette placée au pied d'un mort à la morgue.

Dans les deux premières strophes, le poète joue de la nomination. Il n'est pas encore pleinement inscrit dans la filiation. Il possède la nomination par la mère - le prénom - et il se nomme maison. Cet espace de la maison natale, sorte de ventre de la baleine, est maternel, comme le souligne Bachelard lorsqu'il parle de "la maternité de la maison", « embrassante » telle les bras de la mère (27). Cependant, l'adresse - l'assignation d'une identité placée sur une carte - renvoie davantage à une instance paternelle. S'identifier à un lieu, un point fixe, est l'expression d'un besoin de repères, de stabilité, d'unité. De même, il se décrit à plusieurs reprises tenant la main de sa mère, comme s'il refusait de la lâcher et d'être séparé d'elle ("Holding hands with Mummy », 4, 5). Il expose ainsi son besoin de certitude, d'une conscience de l'être face à un déficit d'être qui se manifeste par ailleurs par le processus d'identification changeante. Or ici, c'est lui qui est maison, même si ce lieu renvoie bien au lien à la mère. Bien qu'il soit tentant de lire ce texte comme l'expression d'un fantasme de retour dans la maison «berceau » (Bachelard 26), voire dans le ventre de la mère, à la lecture de la quatrième strophe, il semble plutôt exprimer l'envie de ramener la mère à lui, de retourner là où il n'a pas à se connaitre et de l'y entraîner :

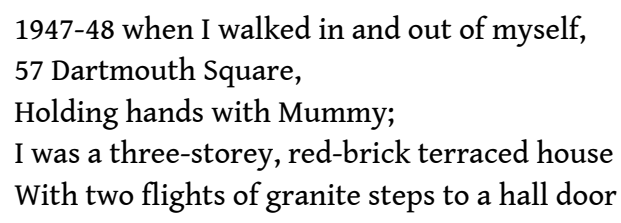


With a recessed porch

In which I never dallied, not even to play in.

I entered only the basement to come in and out of myself [...] (4)

l'évocation de la cave et de la chambre, «basement », « my bedroom », 4, 6), il s'approprie complètement la maison ${ }^{2}$. Le fait qu'il entre et sorte de ce lieu auquel il s'identifie en tenant la main de la mère, semble être non pas l'expression d'un fantasme de régression intra-utérin mais une image qui est plus proche de celle de l'avalement, pour se l'approprier, la posséder. Cette image s'inscrit dans un poème qui peut être lu, d'un point de vue psychanalytique, comme expression d'un désir de retour à un plan pré-psychique, d'avant le stade du miroir, à un état de fusion/confusion avec la mère. Selon Freud, cet état est toujours à l'origine du désir de l'homme et c'est de la nostalgie de cet état que naît le processus de sublimation et l'œuvre d'art (voir Vandermersch in Chemama 102-104). Le poème serait alors «l'objet qui dans la sublimation vient à la place de la Chose ", la mère, le Paradis perdu-cherché, la Chose étant définie comme: «Objet de l'inceste. Ce qu'il y a de plus intime pour un sujet, quoiqu'étranger à lui, structurellement inaccessible, signifié comme interdit (inceste) et imaginé par lui comme le souverain Bien : son être même » (Vandermersch, 102).

En effet, ce poème peut être lu à la fois comme l'expression de la perturbation à l'Autre et comme ce qui permet la triangulation œdipienne, l'ouverture vers le social. Il est le support extérieur de tourments intérieurs : un miroir qui lui permet de se connaître et de créer le lien manquant, défaillant avec le monde. Il est formulation du lien indestructible à la mère, du deuil impossible de ce qui a été son rapport à la mère, mais aussi d'un manque par rapport au père qui n'a pas ici de place et ne participe pas à la séparation.

un premier temps, si la maison natale et l'hiver de ses trois ans sont présentés comme un espace-temps parfait, ce n'est pas tant en raison d'un lieu en soi, mais de la présence de la mère doublée de l'absence du père en ce lieu : «1947-48 there was myself and my mother, / My father gone away for a long time» (4). En effet, ce dernier ne réapparait d'abord que ponctuellement lors des week-ends et il n'est pas perçu comme la personne inquiétante et violente qu'il paraît être dans « First Mixed Party » ou " The Poet and the Judge » dans The Days of Surprise (10-13). Dans The Laughter of Mothers, ouvrage en hommage à sa mère, cette même adresse, Dartmouth Square, renvoie au lieu où sa mère vit en recluse ("a recluse ») en raison du père de Durcan dans "First Place in Ireland" (107-110)3. Dans The Days of Surprise, le vers suivant de « First Mixed Party " y fait écho: "My mother in the cold dark kitchen to which she had been consigned in perpetuity " (12). Ici, l'absence du père lui permet de s'approprier complètement la mère, ce qui génère une impression de perfection apparente. Cependant, cette relation duelle exclusive est également ce qui entraîne l'instabilité, la quête de sa place dans le monde, et de l'homme/des hommes dans le poème. Ce lien défaillant avec le père - l'absence perçue - est ce qui éveille la quête du "Nom-du-Père » pour reprendre le terme lacanien (voir Chemama 390-392). Cette quête est perceptible par l'entremise des nombreux hommes qui parcourent le texte : père, officiers de l'armée britannique, livreurs de charbon et de journaux, Jésus, conducteur de péniche, Dr Roger Bannister, policier, ami du père, Père Noël, Rois Mages.

16 Puisque Durcan donne voix dans ce poème aux fantasmes de l'enfant, des fantasmes archaïques qu'il se réapproprie adulte par le biais de ses écrits, cela crée des effets de brouillage. Le poète semble jouer avec les notions liées à la construction psychologique 
type de l'enfant, laquelle conduit à l'émergence progressive du psychisme : de la fusion du maternage à la fin de l'œedipe en passant par la séparation, avec le stade du miroir (entre six mois et deux ans). Cette émergence se clôt par l'intériorisation du Nom-du-Père, quand le conflit imaginaire se résout par l'intégration symbolique de la fonction du père (voir Chemama 410-414). Cela permet l'inscription définitive dans la filiation et la chaîne de la socialisation. Ici, de manière paradoxale, le poète se décrit adulte à deux reprises comme un «névrosé heureux» ("a happy neurotic», 6, 8). Il énonce son refus de la séparation d'avec la mère et une forme de rejet du père: une triangulation incomplète que l'œuvre vient ici achever, et qui aboutit à l'ouverture sur le monde lors de la scène finale avec les Rois Mages. Il revient à un stade qui se situerait à la frontière de l'ÆEdipe, se positionnant là où il n'y a pas de stabilité définitive mais instabilité, régression possible au sein d'une relation duelle primitive, et recours à des identifications imaginaires idéalisées et/ou changeantes.

Ainsi, Durcan ne dresse pas seulement une série d'autoportraits, mais aussi un portrait de famille partiel. Il y a un manque qui va être compensé par les hommes nommés et/ou décrits au fil du texte. Il dépeint une mère et l'enfant, sorte de résurgence des représentations de la Vierge à l'Enfant avec la répétition d'expressions comme «myself and my mother » (4), « Holding hands with Mummy $(4,5)$, « Mummy and I ( 5 ). Cette impression est renforcée par l'assimilation du poète au Christ, dont la figure surgit d'abord sous les traits d'une péniche puis de l'homme à sa barre lors des promenades en compagnie de sa mère :

We'd climb up wide steps, Mummy and I,

And stand on the towpath to watch the barge

Inside the lock gates rising up

Like Jesus in the Resurrection story

Until the barge achieved its glory and began to chug

Between the banks, its cargo under tarpaulin,

A man in a cloth cap at the tiller, smoking a cigarette

A man's bicycle thrown down at his feet on the deck,

All handlebars, crossbar, raw naked leather saddle [...] (5)

Le texte oscille entre stase et mouvement. Il se sature à nouveau de picturalité par le biais de l'introduction du verbe de vision ("watch ») ainsi que par l'utilisation de formes en ING et de formes nominales qui provoquent un effet de suspension du temps. Avec la description de la péniche qui s'élève et atteint sa gloire (" achieved its glory ») ${ }^{4}$ puis de l'homme à la barre avec à ses pieds un vélo - objet issu d'une réalité brute et nue et non un adorateur ou sa croix (même si la croix apparaît de manière indirecte par le terme " crossbar ", nous soulignons) - ce passage déconstruit et réécrit les représentations du Christ en gloire Ressuscité. Le thème de la Résurrection vient aussi renforcer l'idée de résurgence qui est à l'œuvre tout au long du poème: la résurrection du passé perdu, cherché, retrouvé au travers des mots et des images textuelles. Cet extrait préfigure l'autoportrait où il devient cet homme et où il s'associe de manière indirecte au Christ, avant de se présenter comme une sorte de nouveau Christ attendant à son tour la venue des Rois Mages («Waiting for the Three Wise Men to find me », 6) :

Much as I enjoyed being 57 Dartmouth Square,

My name, role and function as 57 Dartmouth Square,

One day I'd cease to be 57 Dartmouth Square,

Becoming instead the Barge Man at the Tiller

In a Cloth Cap, smoking a cigarette.

It was my fate to be a happy neurotic. (6) 

dans l'effet titre de tableau produit par l'utilisation des italiques et des majuscules. Son changement de statut - ce passage de l'être au devenir - est préfiguré par les mouvements au sein et hors de la maison. C'est comme si une image se transformait en une autre, comme dans un rêve, puisqu'un élément d'une image est à chaque fois repris dans la suivante. Cette représentation de lui-même en conducteur de péniche christique est aussi préparée par les comparaisons avec les travailleurs entrant et sortant de la maison et qu'il relie à cette dernière par des notations telles que : « All twenty-one stone of him " ou "His yellow lined face" (5). La notation de poids et les rides font indirectement réapparaître la façade de la maison et l'évocation de la couleur des cheveux du livreur de journaux ("His tar-black streaky hair», 5) le rattache au charbonnier.

Lorsque le poète s'identifie de manière répétée au Christ, et notamment lorsqu'il se dépeint attendant les Rois Mages (6), il fait de l'expérience de sa présence au monde un jeu sur la Présence. L'Épiphanie est la fête chrétienne qui célèbre la présentation de Jésus aux Rois Mages, leur adoration du Messie, et par là même, son incarnation et sa manifestation, ainsi que sa rencontre avec le monde dans toute sa diversité. C'est comme si le poète s'était lui aussi manifesté, avait ressenti son appartenance au monde et attendait à son tour l'adoration des Rois. Ceci débouche, dans la deuxième partie du poème, sur une déconstruction et une réécriture de L'Épiphanie par le biais d'un jeu sur le sens de ce terme qui s'opère à travers l'évocation de souvenirs.

21 Gombrecht définit l'épiphanie comme la tension entre présence et signification et leur simultanéité («the simultaneity, tension, and oscillation between meaning and presence ", 111). Il souligne à la fois son caractère éphémère, sa dimension spatiale, et la possibilité de décrire sa temporalité comme celle d'un événement (111). Ce que le poète évoque, ce sont des événements de sa vie, marquants, datables et localisables, où il a eu des prises de conscience soudaines et où ce qui était caché est devenu manifeste. Or, les deuxième et troisième souvenirs mettent en relief des formes de violence physique et psychologique. Ce sont des épiphanies douloureuses au cours desquelles il prend conscience de l'aspect dangereux et sombre du monde et des êtres qui l'entourent : «A shocking epiphany of my child's accumulating horror / At the cracks-no, the chasms-in the social fabric of Dublin » (7). Le sens du premier moment diffère. Ce n'est pas tant une épiphanie qu'une réaction vive face à un fait de l'actualité : quand, en 1954, le Dr Roger Bannister a été le premier athlète à couvrir «le mile", une course de demi-fond, en moins de quatre minutes. Ce record déclenche des tentatives répétées d'imitation, de transposition dans son univers, dans les rues autour de Dartmouth Square et le docteurathlète devient un modèle, une figure paternelle de substitution.

Or, ces temps forts préfigurent tous à leur manière l'épiphanie finale, y compris le premier, par la dimension d'imitation :

Christmas Day was not really Christmas Day,

It was the day of Santa Claus, an eccentric deity

Whom my father's corpulent pal, Judge Charlie Conroy,

Pointed out to me one night catwalking the rooftops.

The real Christmas Day was the Feast of the Epiphany, 6 January,

When at long and dear last in the suspenseful chess game of life

I got to move the Three Wise Men into the Crib.

At last they found me and I wriggled in ecstasy.

How ecstatic also seemed these three exotic refugees, 
Melchior, Caspar and Balthasar.

Hailing from the farthest corners of the universe,

From China, Lithuania, Ethiopia.

On the Feast of the Epiphany

In the hallway of 57 Dartmouth Square

Into the Crib on the hall table

I moved the Three Wise Men into position,

A stick of liquorice dangling from my mouth.

I had become again the happy neurotic I aspired to be. (7-8)

Cette épiphanie se révèle être un souvenir fantasque, une rêverie ou un délire décrit comme un moment d'extase, où Durcan fait à nouveau l'expérience d'une joie intense, mais où les Rois Mages ne sont pas réellement présents puisqu'ils sont des figurines de crèche que le poète positionne. L'effet tableau final par lequel il se dépeint et dans lequel il est à nouveau associé de manière indirecte au Christ, résonne avec l'ekphrasis minimale antérieure dans laquelle il se définissait déjà comme un «névrosé heureux » (6).

Ainsi, Durcan contraste espace-temps de bonheur que l'on voudrait encore posséder, hostilité extérieure, et accueil de l'extérieur. En décrivant de manière picturale différents moments clefs de sa vie et en particulier de son enfance, en "faisant image", il leur « [donne] » une nouvelle « présence » (Nancy 2003, 125-126) et les préserve de l'oubli. « La place vide de l'absent comme place non vide, voilà l'image ", déclare Nancy $(2003,128)$. Le poème qui fait image est ici compris comme «l'objet qui dans la sublimation vient à la place de la Chose », la mère, le Paradis perdu-cherché, tout en «[se signalant] par son caractère d'être Autre Chose " puisque " $\left[l^{\prime}\right]$ art a cette fonction de reproduire ex nihilo du signifiant et donc de la Chose comme perdue et c'est pourquoi il est création" (Vandermersch in Chemama, 102, 104). Voyons maintenant comment, dans «Il Bambino Dormiente » (DS 26-28), septième poème du recueil, Durcan cherche à nouveau la Chose perdue, la mère, et tente cette fois de combler le vide, par l'entremise d'une image, la tempera de Giovanni Bellini Madonna in trono che adora il Bambino dormiente, dont il écrit l' ekphrasis. Analysons comment ce faisant il rejoue l'impossible deuil de la mère, «présente l'absence » (Nancy 2003, 128) tout en rendant à nouveau présent un moment passé dans les bras de sa mère afin de tenter de lutter contre la peur du néant et de se réconcilier avec le présent et l'idée de la mort. 


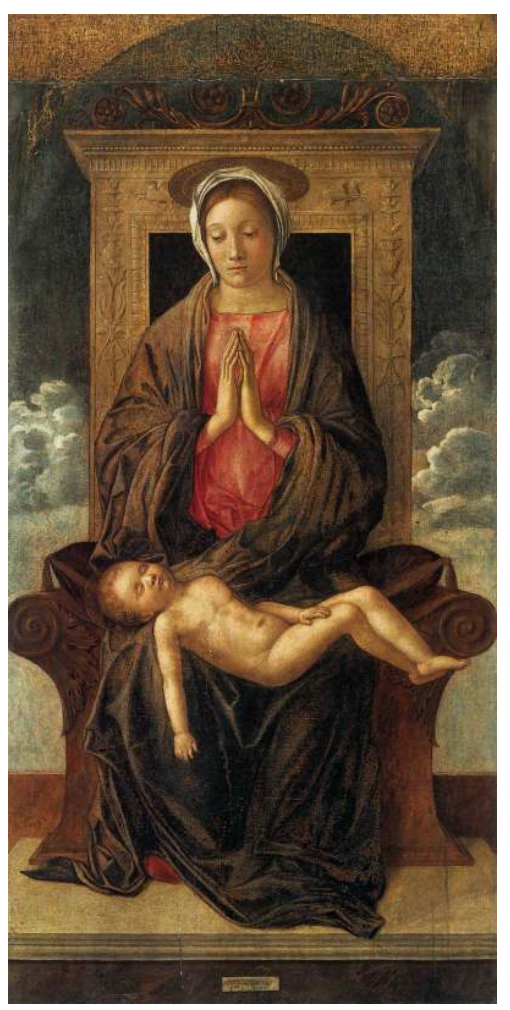

Giovanni Bellini, Madonna in trono che adora il Bambino dormiente (1475), $120 \times 63 \mathrm{~cm}$, tempera sur toile, Gallerie dell'Academia, Venise, Wikimedia Commons

\section{« II Bambino Dormiente » : l'ekphrasis comme moment de rencontre entre art, vie et religion}

Dans «Il Bambino Dormiente », l'ekphrasis comprise dans la strophe centrale du poème est encadrée par les deux strophes relatant le voyage aller et retour de Dublin à Venise dont le but était d'aller voir la tempera de Bellini. Le voyage physique prépare le voyage intérieur dans le temps qui va s'opérer face à l'œuvre. La série de déplacements rapides présentée dans la première strophe de manière factuelle et abrupte ( «I nipped over to Venice ", "Cheap Aer Lingus flight to Marco Polo, / Bus [...] Water bus [...] Half-price entrance fee ", 26) ainsi que la brièveté du voyage ("a day and a night », 26) contraste avec la longueur du moment passé face à l'œuvre, et la minutie avec laquelle elle est décrite en détail. La relation au temps est centrale et c'est le verbe "s'attarder" ( linger ») qui, utilisé en introduction de la seconde strophe et répété en conclusion de cette ekphrasis, crée un effet de cadrage supplémentaire : «I linger alone before Bellini's small picture ", «I linger - I linger all day » (26-27). Ce n'est pas dans le mouvement mais dans une immobilité, mise en abyme, que tout se joue et que naît l'intensité en lien avec l'expérience esthétique: dans la contemplation de la représentation fixe d'un être regardant un autre dormir, immobile.

Ce voyage et ce temps d'arrêt face à l'œuvre sont dictés par un besoin impérieux - mis en relief par la répétition de «need»- de lutter contre un sentiment de dissolution imminente, de solitude et d'aliénation (« On the spit of dissolution, / Estranged from my family ", 26) : 
I needed to see one particular painting in the Gallerie dell'Academia

By Giovanni Bellini [...]

Needed to? Yes-needed to. [...]

I needed to see again

The most affectionate yet sacred family portrait ever painted.

[...] I needed-

As we need to drink water to stave off death-

I needed to see myself as originally I was [...]

I needed to see her slightly prised-open eyes glancing down [...]

I needed to see again with my own eyes [...] (26-27)

C'est la quête d'un bonheur, d'une connexion, voire d'une fusion perdus qui est formulée.

En allant chercher une image qui représente la Vierge, la mère archétypale portant son enfant, et en superposant son image et celle de sa mère sur le Christ et la Vierge de l'œuvre, il fait d'abord ressurgir du temps écoulé. Il le matérialise et le rend à nouveau présent. C'est d'ailleurs sur le mode de la répétition que le poème s'inscrit, comme l'indique les trois utilisations du mot «encore» («again», 9, 27, 29). L'écriture de l' ekphrasis, qui se mêle à la description de son observation de la tempera et à sa projection sur celle-ci, lui permet ensuite de se réapproprier l'œuvre et le passé, de prolonger l'intimité d'autrefois et celle née de la contemplation, et de les fixer sur la page.

Cette expérience du voir - décrite comme besoin impérieux de re-voir, et ainsi de tenter de re-posséder - n'est pas placée du côté de l'avoir, mais de celui du déficit d'être, de l'avoir-été, de l'aliénation, de la perte. Ce qui se joue ici, c'est l'impossible deuil de la mère et la recherche d'un lien qui lui rappelle une certitude d'être en réponse à un sentiment d'aliénation, à la peur du vide, de la mort. L'expérience visuelle au cœur de la quête du poète fait écho à "l'inéluctable modalité du visible (ineluctable modality of the visible) », tel que Joyce l'écrit dans Ulysses ou à "l'inéluctable scission du voir », tel que Georges DidiHuberman la conceptualise en partant de cet extrait de Ulysses où le spectacle de la mer « est regardé par la perte de sa mère » (12) : « la modalité du visible devient inéluctable - c'està-dire vouée à une question d'être - quand voir c'est sentir que quelque chose inéluctablement nous échappe, autrement dit : quand voir, c'est perdre » (14).

C'est une dialectique de la présence et de l'absence qui s'articule. L'œuvre d'art qui "[donne] présence» (Nancy 2003, 126), et qui réapparaît par le biais de l'ekphrasis, renvoie aussi au vide, à ce qui est absent, perdu. Ce qu'il fixe du regard, c'est ce qu'il voudrait fixer, ce qu'il tente de capturer par les mots mais c'est aussi ce qui échappe. Par ce tableau et son ekphrasis, il y a, selon la formule de Didi-Huberman, "trait» et " retrait »; "apparition et disparition", mais aussi réapparition et re-disparition; " cuvre de perte» (13-14), indice de sa propre mort et œuvre de (ou tentative de) reconquête indirecte; travail de deuil - présenté comme impossible - qui semble pourtant s'opérer partiellement puisque le poète exprime une forme d'apaisement, de réconfort apporté par la foi. Ce tableau devient un « objet auratique », " proche et distant à la fois, mais distant dans sa proximité même", pour reprendre l'analyse de DidiHuberman du concept d'aura de Walter Benjamin (104). La visualité est auratique dans le sens où l'apparition de l'objet retranscrit dans l'ekphrasis « déploie [...] ses images [...] pour en poétiser, en ouvrager, en ouvrir l'aspect autant que la signification, pour en faire une œuvre de l'inconscient» où les temps sont «tressés, joués et déjoués, contredits et surdimensionnés » (105).

Par effet miroir, l'œuvre qui est ici «[regardée] par la perte de sa mère » (Didi-Huberman, 12), dépeint la mère face à la perte à venir du fils. Dans l'œuvre de Bellini, le Christ est à la 
fois présenté au spectateur comme un objet de dévotion et un rappel de son sacrifice. Les deux pôles opposés de la vie, la petite enfance et la mort, sont condensés dans une même image par l'entremise de la position de l'enfant Jésus endormi qui reprend celle d'une descente de croix. Tout comme dans le tableau, sommeil et (apparence de) mort sont liés à travers le poème par des notations telles que : "Sleeping the sleep of death [...] his sleeping visage, his tall, thin, grey, aged features », «I needed to see again with my own eyes / Her apprehension of the inevitable» (27). Ceci répond aux vers :

[...] Bellini's small picture

Of all that it means to be your mother's son

In the mortal world, all that it means

To be a young mother doomed. (26)

De plus, comme le souligne Nancy dans sa définition, une dialectique de la présence et de l'absence, qui renvoie à la naissance et à la mort, mais aussi à la Présence, est à l'œuvre dans chaque portrait :

Ce qui est portrait, c'est naissance et mort du sujet, lequel n'est rien d'autre que cela, naissance à la mort et mort à la naissance, ou encore infini rappel à soi. Si le portrait suscite et retient en lui ce rappel et cette anticipation [...] d'une présence égale à son absence, c'est bien parce qu'il est le dépôt, et le suppôt, du basculement du divin dans l'absence [...]. Chaque portrait joue au singulier l'impossible portrait de Dieu [...]. Le portrait rappelle l'icône et lui ressemble comme l'absence de la présence rappelle, pour lui ressembler, la présence de l'absence. Le portrait rappelle en tout un chacun fini l'infinie distension de l'un. (Nancy 2000, 65, 69)

Le poète s'identifie au Christ endormi et fait de sa mère une icône, une nouvelle Vierge ; il la transfigure et lui rend hommage tout en célébrant la beauté de l'œuvre. Il passe de l'histoire au mythe. Il s'approprie ce "portrait de famille sacrée " ("sacred family portrait », DS, 26) et le transforme en «infini rappel » à lui et à sa mère tout en jouant avec la notion de présence/Présence :

I needed to see myself as originally I was:

A naked male infant draped across my mother's knees,

Sleeping the sleep of death;

I needed [...]

To check again that she does indeed have red hair

Parted down in the middle

In a white veil

Under the flat gold plate of her halo

And that her cheeks also are red-

Not with rouge-

But with all

That is most virginal, auroral,

Most purely West of Ireland peasant princess,

Palestinian Jewess,

Her slender fingers craned tall in prayer. (27, nous soulignons)

La superposition de l'image de sa mère et de la Vierge de Bellini se confirme et s'opère de par l'association géographique de l'Ouest de l'Irlande à la Palestine. Il ne la transforme pas en simple icône, mais en la quintessence même de la Vierge par son utilisation des superlatifs. La transfiguration de la mère en particulier et de la femme en général en Vierge est un élément qui parcourt l'œuvre de Durcan où il exprime son obsession pour sa mère, la Vierge et les femmes, superposant parfois de manière incestueuse les images de mère, de la Mère archétypale ou Vierge et de l'amante. Dans " Mother Playing Golf in a Bikini » de The Laughter of Mothers, Durcan écrit: «I am a male for whom there is a mystique / About women and the first woman I ever knew was my mother » (94). Dans 
The Days of Surprise, ce phénomène est par exemple repris dans « The Man of Advancing Years and the Girl on a Bicycle", où il présente chaque femme comme une nouvelle Vierge ou une Vierge en puissance :

Did you not learn [...]

That the girl on a bicycle always takes precedence?

Always the not-yet pregnant Mother of God? (95)

Il montre par ailleurs un intérêt tout particulier pour les (seins des) mère(s) dans « Other Boys' Mothers » ou "The Young Mother on the Country Bus in El Salvador» $(9,42)$. La rousseur de la mère célébrée de manière indirecte à travers l'ekphrasis dans «Il Bambino Dormiente » rappelle les "taches de rousseurs et les cheveux roux» de ses premiers amours d'enfance évoqués dans le second poème du recueil, « First Mixed Party » (11). En outre, dans "Il Bambino Dormiente», si la notation répétée de la couleur rouge ("red hair ", " her cheeks also are red- / Not with rouge- ", 27) évoque à la fois le tableau et la rousseur de la mère, elle peut aussi renvoyer au rouge des représentations de Vierge au chardonneret (rouge d'un des vêtements de la Vierge, de la tache de l'oiseau, des cerises qu'il grignote parfois). Par cette description, les images du chardonneret qui illustrent la couverture, parcourent le recueil, et trouvent ici un écho dans le rappel du sacrifice du Christ à venir, Durcan semble recréer la vision d'une de ces Vierges au chardonneret. C'est cette vision déconstruite et reconstruite qui apparaît en filigrane et se propage à travers The Days of Surprise, par le biais des images de chardonneret et de la mère/Vierge. Dans The Days of Surprise, le poète écrit également les ekphraseis d'autres représentations de la Vierge comme dans "Our Lady of Westport», celle du vitrail de Patrick Pye se trouvant dans l'église catholique de St Mary à Westport, dans le comté de Mayo ou dans "The Days of Surprise», celle de la statue de la Vierge située derrière l'église de Ringsend, dans le quartier où le poète vit actuellement $(43,39)^{5}$. C'est le lien qui l'unissait à sa mère qu'il recherche de manière répétée, voire obsessionnelle, y compris lorsqu'il donne voix à son espoir de retrouvailles après la mort :

I got a Ryanair early flight back to Dublin

To settle my affairs and get ready for my own little sleep,

Meeting my mother in the big deep. (28)

C'est cette foi que le poète formule à la fin de «Il Bambino Dormiente » et dans le poème qui semble en être la continuité : « Meeting Mother in the Big 0 » (29). De plus, ce dernier se conclut sur une citation de l'évangile selon saint Luc $(2: 35)$ : «And a sword shall pierce through your own soul » (29). Ce passage correspond à l'annonce à la Vierge de la mort du Christ et du terrible chagrin à venir, afin qu'elle puisse s'y préparer. Il entre en résonance avec le destin de la mère (" doomed ») et avec la mort (du Christ/poète) annoncée dans « Il Bambino Dormiente » (26-28).

Dans «Il Bambino Dormiente », c'est grâce à la contemplation du tableau - puisque l'image fait ressurgir un temps écoulé tout en confrontant le poète avec sa propre mort - que ce dernier se console et se réconcilie à la fois avec le présent et sa propre finitude. La répétition de l'expression « all that it means » (26) souligne l'aspect hautement signifiant de l'expérience esthétique. Cette réconciliation a une dimension religieuse et s'inscrit dans l'espoir d'une Résurrection à venir. Elle a aussi une conséquence humaine immédiate. Durcan unit profane et sacré, et ce dès sa désignation du tableau : «The most affectionate yet sacred family portrait ever painted » (26). De même, dans son ekphrasis du vitrail représentant la Vierge dans "Our Lady of Westport ", il conclut en abolissant la distinction entre sacré et profane : "That woman would make you want to be human » 
(43). En observant la tempera de Bellini, pourtant seul dans cette salle (" alone », 26), il revit un instant de tendresse partagé, éprouve un sentiment d'appartenance. Cela lui permet ensuite de recréer du lien, des formes de connexion avec les autres, là où il n'y avait avant que solitude et aliénation. Comme par effet de conséquence, il retrace dans la strophe finale ses échanges avec le concierge de la pension où il passe la nuit, et sa rencontre avec Ya :

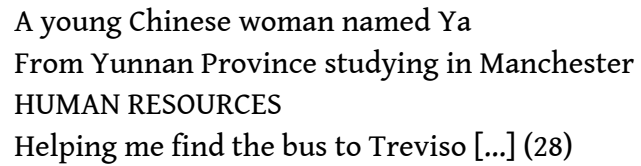

Cette figure féminine qui condense la diversité du monde, symbolise son ouverture sur le social et rappelle ainsi les Rois Mages du premier poème. Outre l'aide qu'elle lui apporte, elle est celle qui symbolise et lui rappelle ses propres « RESSOURCES HUMAINES ». Or ces rencontres ne sont possibles que parce que l'expérience esthétique, ce moment face à l'œuvre, lui a permis de retrouver à la fois un lien avec l'humanité, une place (momentanée ?) dans le monde, et de se réconcilier avec le destin qui attend tout homme, la mort.

Durcan exprime son impossible deuil du rapport à la mère et son besoin d'appartenance, de lien. Il nous parle de vie et de mort, de présence et de perte, mais aussi de tentative de capture d'instants passés, d'enchantement. Dans "The Butterfly Collector of Corofin ", il donnait voix à un désir de régression, à une peur de la mort et d'un enchantement qui deviendrait une fixité mortifère :

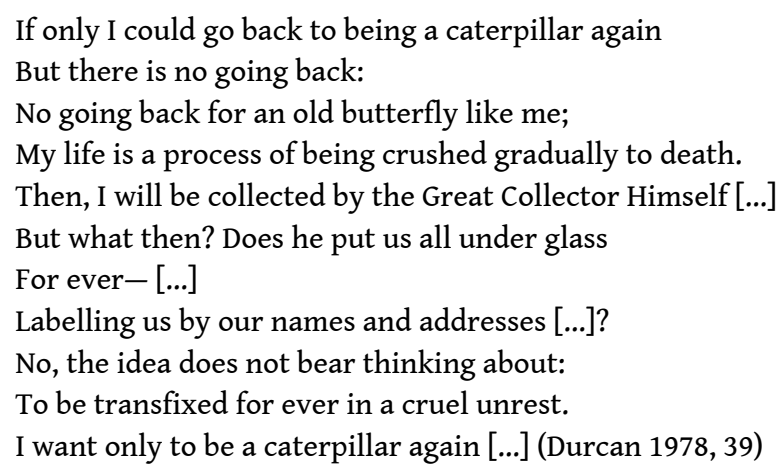

Cette peur est transcendée à travers son œuvre par la recherche d'un enchantement de vie qui intégrerait le mouvement et le changement. Ici, il manifeste à nouveau un désir de régression mais ne rejette plus cet étiquetage : c'est lui qui s'attache au pied l'adresse de sa maison d'enfance, paradis terrestre perdu. Il accepte et semble embrasser une forme de fixité en recherchant un repère, un élément stable comme la maison. Rêvant à la maison natale, il revient « au pays de l'Enfance Immobile, immobile comme Immémorial » et écrit "des fixations de bonheur", pour reprendre la formule de Bachelard (25). Cependant, il oscille entre immobilité et mouvement. Il crée dans l'espace poétique un temps suspendu, surdimensionné, complexe, tressé, où l'intensité naît de la stase, mais il écrit également le changement - notamment par l'entremise de métamorphoses - et accepte le flux de la vie. Il exprime sa foi en un au-delà, s'y projette et répète son désir d'y retrouver sa mère. Ce faisant, il déconstruit et reconstruit à nouveau le mythe chrétien car, dans ce Paradis auquel il aspire, il se place aux côtés de la Mère, et non du Père. 


\section{BIBLIOGRAPHIE}

Bachelard, Gaston. La Poétique de l'espace. Paris : PUF, 1957.

Chemama, Roland et Bernard Vandermersch. Dictionnaire de la Psychanalyse. Paris : Larousse, 2009.

Durcan, Paul. Sam's Cross. Dublin: Profile poetry, 1978.

Durcan, Paul. The Days of Surprise. London: Harvill Secker, 2015.

Durcan, Paul. The Laughter of Mothers. London: Harvill Secker, 2007.

Gombrecht, Hans Ulrich. Production of Presence. What Meaning Cannot Convey. Stanford: Stanford UP, 2004

Didi-Huberman, Georges. Ce que nous voyons, ce qui nous regarde. Paris : Éditions de Minuit, 1992.

Nancy, Jean-Luc. Le Regard du portrait. Paris : Galilée, 2000.

Nancy, Jean-Luc. Au fond des Images. Paris : Galilée, 2003.

Wallace, Armita. « Paul Durcan: The Most Playful Poet in Ireland». The Irish Times (17 mars 2015). http://www.irishtimes.com/culture/books/paul-durcan-the-most-playful-poet-inireland-1.2138673 (dernière consultation le 11 mai 2016).

\section{NOTES}

1. Chardon qui apparait sur la couverture de The Days of Surprise.

2. Le choix des différents espaces de la maison vient aussi renforcer la dialectique du dedans et du dehors, le porche, la porte du hall d'entrée et la fenêtre de la chambre étant des endroits frontières entre intérieur et extérieur.

3. Voir également la référence à l'internement du poète et à la reaction de la mère (« When her husband the judge had their nineteen-year-old son, / In the Spring of 1964 / Committed to St John of God mental hospital / She was not consoled, but she was helpless ») et la dimension effrayante du père (" afraid of my husband the Judge », Durcan 2007, 84, 103).

4. L'élévation de la péniche qui fait écho au corps du Christ lévitant hors de son tombeau est sur un plan horizontal, contrairement aux représentations traditionnelles où l'on peut observer le Christ émergeant du tombeau, triomphant, en gloire, sur un mode vertical.

5. Ces précisions par rapport à la localisation des œuvres nous ont été apportées par le poète lors d'une communication privée dans un courriel du 27 juillet 2015. 


\section{RÉSUMÉS}

Dans « 57 Dartmouth Square» et «Il Bambino Dormiente», Paul Durcan s'auto-représente, notamment seul ou aux côtés de sa mère, entre avoir-été, être et devenir, immobilité et mouvement. Ce faisant, il tresse et condense temps, histoire et mythe, au sein d'images textuelles. Il écrit sa nostalgie d'un lien, voire d'une fusion avec la mère. Par le biais de l'écriture picturale qui peut découler d'une expérience esthétique, il rend à nouveau présents des moments de bonheur perdus et les prolonge, tout en donnant voix à une dialectique de la présence et de l'absence qui peut se faire jeu sur la Présence. Cependant, l'espace poétique n'est pas uniquement espace enchanté où une forme de résurrection s'opère. Il est aussi l'endroit où s'exprime pour le poète la quête de sa place dans le monde et d'un lieu de réconfort où il se réconcilie avec le présent et l'avenir.

In "57 Dartmouth Square" and "Il Bambino Dormiente", Paul Durcan depicts himself, alone or with his mother, and oscillates between past, being and becoming - between stillness and movement. In so doing, he weaves different times and temporalities together, unites history and myth, and encapsulates them within textual images. He expresses his nostalgia and yearning for a lost link, for his fusion with his mother. Through a form of pictorial writing born out of an aesthetic experience, he brings back to life happy moments thought to be lost. He extends them while giving voice to a dialectics of presence and absence, which involves a play on the notion of Presence. Nonetheless, these poems are not only the enchanted space where a form of resurrection of the past becomes possible. They are also the space where the poet searches for his place in this world and where he reconciles himself with his present situation and his future.

\section{INDEX}

Keywords : stillness, presence, loss, belonging, portrait, self-portrait, moment, movement Mots-clés : immobilité, présence, perte, appartenance, portrait, autoportrait, moment, mouvement

\section{AUTEURS}

\section{CATHY ROCHE-LIGER}

Cathy Roche-Liger est l'auteur de la première thèse française sur le poète irlandais Paul Durcan («Poétique du pictural dans l'œuvre de Paul Durcan », 2011). Rattachée à l'équipe B1 du FoReLL de l'Université de Poitiers, elle a écrit plusieurs articles sur P. Durcan, notamment sur ses ekphraseis, et également travaillé sur les poètes W.H. Auden, Michael Longley, John Montague, Harry Clifton, Trevor Joyce et Geoffrey Squires. Elle s'intéresse particulièrement à la poésie irlandaise contemporaine et aux interactions entre le visuel et le verbal. 